\title{
Dietary water affects human skin hydration and biomechanics
}

This article was published in the following Dove Press journal:

Clinical, Cosmetic and Investigational Dermatology

3 August 2015

Number of times this article has been viewed

\author{
Lídia Palma' \\ Liliana Tavares Marques' \\ Julia Bujan 2,3 \\ Luís Monteiro Rodrigues ${ }^{1,4}$ \\ 'CBIOS - Research Center for \\ Health Science and Technologies, \\ Universidade Lusófona, Campo \\ Grande, Lisboa, Portugal; '2Department \\ of Medicine and Medical Specialities, \\ Universidad de Alcalá de Henares, \\ Madrid, Spain; ${ }^{3}$ CIBER-BBN, Madrid, \\ España, Spain; ${ }^{4}$ Department of \\ Pharmacological Sciences, School \\ of Pharmacy, Universidade de \\ Lisboa, Lisboa, Portugal
}

\begin{abstract}
It is generally assumed that dietary water might be beneficial for the health, especially in dermatological (age preventing) terms. The present study was designed to quantify the impact of dietary water on major indicators of skin physiology. A total of 49 healthy females (mean $24.5 \pm 4.3$ years) were selected and characterized in terms of their dietary daily habits, especially focused in water consumption, by a Food Frequency Questionnaire. This allowed two groups to be set - Group 1 consuming less than 3,200 mL/day ( $\mathrm{n}=38)$, and Group 2 consuming more than 3,200 $\mathrm{mL} /$ day $(\mathrm{n}=11)$. Approximately $2 \mathrm{~L}$ of water were added to the daily diet of Group 2 individuals for 1 month to quantify the impact of this surplus in their skin physiology. Measurements involving epidermal superficial and deep hydration, transepidermal water loss, and several biomechanical descriptors were taken at day 0 (T0), 15 (T1), and 30 (T2) in several anatomical sites (face, upper limb, and leg). This stress test ( 2 L/day for 30 days) significantly modified superficial and deep skin hydration, especially in Group 1. The same impact was registered with the most relevant biomechanical descriptors. Thus, in this study, it is clear that higher water inputs in regular diet might positively impact normal skin physiology, in particular in those individuals with lower daily water consumptions.
\end{abstract}

Keywords: dietary water, water consume, skin hydration, TEWL, skin biomechanics

\section{Introduction}

Water is a large component of the human body and plays a key role in normal physiological balance. This general concept has been widely explored by the food/beverage market to cultivate the idea that an increase of water intake in our diet might act as a health (and antiage) promotor. Regarding skin health, this association between water and better (skin) performance has been widely accepted, although not clearly demonstrated. ${ }^{1-3}$

Water is the main component of cells and tissues, a major element of body fluid compartments, ${ }^{4}$ and represents $75 \%$ and $60 \%$ (from birth and in adults, respectively) of body's composition. It is an essential nutrient with unique properties as a solvent for ionic compounds and solutes ${ }^{5}$ and acts as a carrier with a central role in cell homeostasis. ${ }^{6,7}$ Water is the environment in which all transport systems work. ${ }^{8}$ It helps in maintaining body volume (intracellular and extracellular), ${ }^{9,10}$ which is essential to prevent dehydration, a potentially life-threatening condition. ${ }^{11}$ The water in the body also plays an important role in thermoregulation ${ }^{10}$ and acts as a lubricant and shock absorbent. ${ }^{10}$

Several studies have suggested that the amount of water supplied by regular food and beverages, including the water produced by the cellular metabolism, is not sufficient
Correspondence: Luís Monteiro Rodrigues CBIOS- Research Center for Health Science and Technologies, Universidade Lusófona, Campo Grande, 376 1749-024 Lisboa, Portugal. Email monteiro.rodrigues@ulusofona.pt 
to meet the body's daily water requirements, ${ }^{10,12-14}$ even considering the very effective neuroendocrine osmoreflexes regulating thirst and voluntary water ingestion. ${ }^{13-15}$ Unlike other essential nutrients, daily recommendations for water consumption are often disregarded, and a clear definition of the daily water requirement needs does not exist. However, there are some indicative recommendations. The "Dietary Guidelines for Americans 2010" report ${ }^{15}$ establishes as adequate water intake 3.7 and $2.7 \mathrm{~L}$ /day for men and women, respectively, between 19 and 30 years old, while the European Food Safety Authority (EFSA) indicates dietary reference values of 2.0 and $2.5 \mathrm{~L}$ of water per day for women and men, respectively. ${ }^{16}$

Regarding human skin's physiology, cutaneous water content is known to play an important role in different skin functions, such as the water "barrier" function or the "envelope" function, and water deficiency is associated with several dermatological dysfunctions. ${ }^{17-19}$ Nevertheless, a direct relationship between these properties and regular dietary water consumption have not been clearly demonstrated, and only very few publications have addressed this theme. ${ }^{20,21}$

In this study, a validated Food Frequency Questionnaire (FFQ) was used to measure the total water intake in a population, ie, the water they drink, the water content of the dietary nutrients, and the water produced by regular metabolism. ${ }^{22}$ In this way, a relationship between some cutaneous functions and water ingestion could be measured in order to look deeper into the impact of dietary water on normal skin physiology.

\section{Material and methods}

\section{Subjects}

A convenience sample of 49 healthy female volunteers, aged between 22 and 34 (24.5 \pm 4.3$)$ years was selected. The effects of gender on normal physiology, including the skin are known. ${ }^{22-26}$ So, this research compromise was intended to reduce the gender-related variability. The selection of volunteers took place after informed written consent was obtained, in accordance with previously established inclusion criteria. The methodologies used fully complied with all ethical standards set by the Declaration of Helsinki and its amendments, ${ }^{27}$ and were previously approved by the institution's ethical commission.

\section{Experimental design}

The population's water consumption pattern involved all contributions accounting for the total amount of water consumed. The measurement instrument was an FFQ, which was previously validated to the Portuguese population. ${ }^{22}$
Volunteers were grouped according to their total water consumption, which corresponded to the sum of drinking water (DrW), water from the dietary nutrients (DiW), and water produced by metabolism (MeW). ${ }^{22}$ Two groups with different total daily water consumptions were identified by cluster analysis (see the following section) as Group 1, corresponding to a total water consumption lower than 3,200 $\mathrm{mL} /$ day $(\mathrm{n}=38)$, and Group 2, corresponding to a water total consumption higher than $3,200 \mathrm{~mL} /$ day $(\mathrm{n}=11)$. The groups were stratified by the total water consumption over the preceding 4 weeks, with ratings on day 0 (T0), on day 15 (T1), and on day 30 (T2). Volunteers were then asked to supplement their normal diet with a fixed daily amount of water for 4 weeks in order to establish the impact of this surplus on their normal skin physiology. A mean value of 2,000 $\mathrm{mL}$ water/day was adopted as the reference surplus, based on the dietary reference values for women from EFSA. ${ }^{16}$

To quantify skin functions, measurements took place after full acclimation to room conditions by volunteers (temperature and humidity $21^{\circ} \mathrm{C} \pm 1{ }^{\circ} \mathrm{C}, 45 \% \pm 5 \%$, respectively) for a period of approximately 20 minutes. All evaluations were conducted in the absence of heat sources and forced convection, according to previously published recommendations. ${ }^{23}$ Five anatomical sites were measured - face (zygomatic and forehead), arm (ventral forearm and hand), and leg (external face). BMI (body mass index) and blood pressure were controlled throughout the study in order to identify potential changes in weight and hemodynamics. The BMI was measured by the Quetelet's formula, while hemodynamics were monitored by blood pressure and heart rate measurements.

\section{Biometrics}

The epidermal "barrier" function was assessed by the transepidermal water loss (TEWL) measurement, obtained with

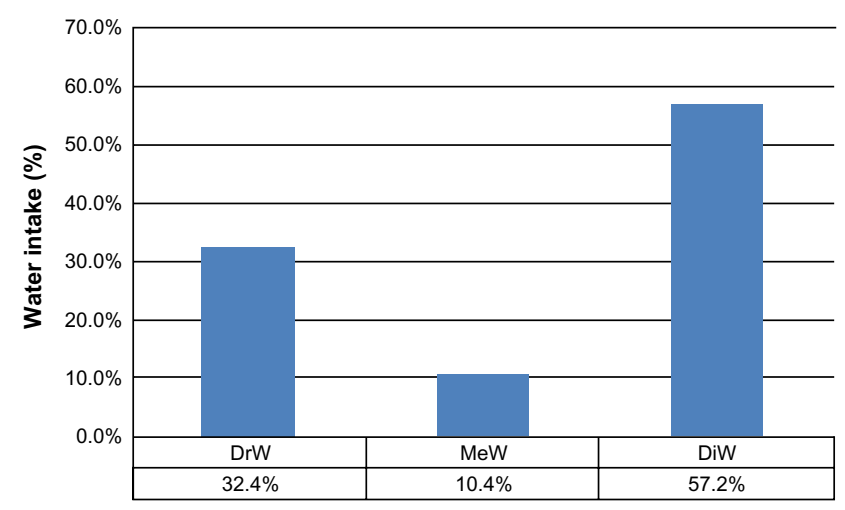

Figure I Contributions of $\mathrm{DrW}, \mathrm{MeW}$, and $\mathrm{DiW}$ to total water intake. Abbreviations: DrW, drink water; MeW, metabolic water; DiW, diet water. 
Table I Transepidermal water loss changes detected as an indicator of epidermal "barrier" in different anatomical areas in both groups of volunteers after the diet water surplus

\begin{tabular}{|c|c|c|c|c|c|c|}
\hline & \multirow[t]{2}{*}{ T0 (mean \pm SD) } & \multirow[t]{2}{*}{ TI $($ mean \pm SD $)$} & \multirow[t]{2}{*}{ T2 (mean \pm SD) } & \multicolumn{3}{|l|}{$P$-value } \\
\hline & & & & (TI vs T0) & (T2 vs TI) & (T2 vs T0) \\
\hline \multicolumn{7}{|l|}{ Group I } \\
\hline Forehead & $10.84 \pm 3.88$ & $9.33 \pm 3.52$ & $10.46 \pm 2.96$ & $0.04 I^{*}$ & 0.176 (ns) & $\mathrm{I} .000$ (ns) \\
\hline Zygomatic & $10.55 \pm 3.40$ & $9.75 \pm 3.17$ & $9.88 \pm 3.02$ & $0.563(\mathrm{~ns})$ & $0.80 I$ (ns) & $1.000(n s)$ \\
\hline Hand & $8.88 \pm 3.25$ & $8.31 \pm 3.01$ & $8.40 \pm 2.77$ & $0.64 \mathrm{I}(\mathrm{ns})$ & 1.000 (ns) & 0.957 (ns) \\
\hline Forearm & $6.14 \pm 2.34$ & $5.55 \pm 1.83$ & $5.79 \pm 1.64$ & $0.346(\mathrm{~ns})$ & 1.000 (ns) & 1.000 (ns) \\
\hline Leg & $5.88 \pm 2.12$ & $5.74 \pm 1.53$ & $6.57 \pm 1.94$ & 1.000 (ns) & 0.080 (ns) & 0.173 (ns) \\
\hline \multicolumn{7}{|l|}{ Group 2} \\
\hline Forehead & $10.98 \pm 4.05$ & $9.39 \pm 4.02$ & $9.76 \pm 3.56$ & 0.406 (ns) & 1.000 (ns) & 1.000 (ns) \\
\hline Zygomatic & $10.00 \pm 2.97$ & $8.97 \pm 4.29$ & $9.56 \pm 3.42$ & 1.000 (ns) & 1.000 (ns) & 1.000 (ns) \\
\hline Hand & $8.87 \pm 2.98$ & $7.62 \pm 2.43$ & $8.84 \pm 4.21$ & $0.612(\mathrm{~ns})$ & 1.000 (ns) & 1.000 (ns) \\
\hline Forearm & $5.83 \pm 1.33$ & $4.98 \pm 1.95$ & $4.90 \pm 1.39$ & $0.702(\mathrm{~ns})$ & 1.000 (ns) & $0.136(\mathrm{~ns})$ \\
\hline Leg & $5.35 \pm 1.48$ & $4.7 I \pm I .33$ & $5.3 \mathrm{I} \pm 1.57$ & $0.858(\mathrm{~ns})$ & $\mathrm{I} .000(\mathrm{~ns})$ & $\mathrm{I} .000$ (ns) \\
\hline
\end{tabular}

Notes: Values are expressed in $\mathrm{g} / \mathrm{m}^{2} / \mathrm{h}$. $* \mathrm{p}<0.05$.

Abbreviations: ns, not significant; SD, standard deviation; T0, day 0; TI, day I5; T2, day 30; vs, versus.

the Tewameter TM 300 (CK Electronics, Cologne, Germany), $\quad$ the Cutometer CM 575 system (in mm) from CK Technologies. expressed in $\mathrm{g} / \mathrm{m}^{2} / \mathrm{h}$, and by the epidermal hydration measured The utility descriptors chosen were extensibility $\left(U_{\mathrm{f}}\right)$, the ability by the MoistureMeter SC and Moisturemeter D (Delphin Tech- of the skin to return to its original state $\left(U_{\mathrm{f}}-U_{\mathrm{a}}\right)$, total elasticity nology D, Bergisch Gladbach, Germany) system, expressed in $\quad\left(U_{\mathrm{a}} / U_{\mathrm{f}}\right.$, including pulse stretching and recovery $)$, elastic function $\mathrm{AU}$ (arbitrary units). The "envelope" function was assessed by $\quad\left(U_{\mathrm{r}} / U_{\mathrm{e}}\right)$, and viscoelasticity $\left(U_{\mathrm{v}} / U_{\mathrm{e}}\right)^{24,28,29}$

Table 2 Skin hydration changes detected in different anatomical areas in both groups of volunteers after the dietary water surplus

\begin{tabular}{|c|c|c|c|c|c|c|}
\hline & T0 (mean \pm SD) & TI (mean \pm SD) & T2 (mean \pm SD) & $\begin{array}{l}\text { P-value } \\
\text { (TI vs T0) }\end{array}$ & $\begin{array}{l}P \text {-value } \\
\text { (T2 vs TI) }\end{array}$ & $\begin{array}{l}P \text {-value } \\
\text { (T2 vs T0) }\end{array}$ \\
\hline \multicolumn{7}{|c|}{ Surface hydration } \\
\hline \multicolumn{7}{|c|}{ Group I } \\
\hline Forehead & $53.97 \pm 22.44$ & $65.24 \pm 17.03$ & $75.52 \pm|4.3|$ & $0.000 * *$ & $0.000 * *$ & $0.000 * *$ \\
\hline Zygomatic & $48.66 \pm 25.4 I$ & $57.04 \pm 23.93$ & $69.52 \pm 21.51$ & $0.025^{*}$ & $0.000 * *$ & $0.000 * *$ \\
\hline Hand & $33.59 \pm 18.29$ & $30.31 \pm 16.91$ & $47.48 \pm 15.60$ & 0.125 (ns) & $0.008^{*}$ & $0.000 * *$ \\
\hline Forearm & $29.09 \pm 11.77$ & $35 .|4 \pm| 3.7 \mid$ & $37.83 \pm \mid 3.01$ & $0.040^{*}$ & $0.180(\mathrm{~ns})$ & $0.000 * *$ \\
\hline Leg & $34.85 \pm 17.94$ & $41.11 \pm 13.53$ & $45.81 \pm 16.68$ & $0.036^{*}$ & 0.172 (ns) & $0.000 * *$ \\
\hline \multicolumn{7}{|l|}{ Group 2} \\
\hline Forehead & $50.64 \pm 18.77$ & $49.67 \pm 15.60$ & $61.86 \pm 17.83$ & $\mathrm{I} .000$ (ns) & $0.023^{*}$ & 0.176 (ns) \\
\hline Zygomatic & $38.76 \pm 23.02$ & $47.77 \pm 21.13$ & $55.70 \pm 25.95$ & 0.301 (ns) & 0.601 (ns) & 0.126 (ns) \\
\hline Hand & $27.01 \pm 10.06$ & $36.08 \pm 13.52$ & $37.26 \pm 13.50$ & 0.312 (ns) & $\mathrm{I} .000$ (ns) & 0.238 (ns) \\
\hline Forearm & $26.89 \pm 22.16$ & $28.16 \pm 12.30$ & $27.94 \pm 7.85$ & $\mathrm{I} .000$ (ns) & $\mathrm{I} .000$ (ns) & $\mathrm{I} .000$ (ns) \\
\hline Leg & $33.05 \pm 19.57$ & $34.34 \pm 12.77$ & $32.64 \pm 11.66$ & $\mathrm{I} .000$ (ns) & $\mathrm{I} .000$ (ns) & 1.000 (ns) \\
\hline \multicolumn{7}{|c|}{ Deep hydration } \\
\hline \multicolumn{7}{|c|}{ Group I } \\
\hline Forehead & $29.20 \pm 4.88$ & $36.34 \pm 9.25$ & $40.62 \pm 10.21$ & $0.004^{*}$ & $0.008^{*}$ & $0.000 * *$ \\
\hline Zygomatic & $29.25 \pm 4.55$ & $35.30 \pm 9.06$ & $39.58 \pm 10.63$ & $0.012^{*}$ & $0.007^{*}$ & $0.000 * *$ \\
\hline Hand & $33.5 I \pm 5.45$ & $41.29 \pm 10.47$ & $46.02 \pm 11.95$ & $0.004^{*}$ & $0.03 I^{*}$ & $0.000 * *$ \\
\hline Forearm & $23.32 \pm 3.96$ & $28.37 \pm 7.50$ & $30.64 \pm 8.65$ & $0.030^{*}$ & $0.136(\mathrm{~ns})$ & $0.000 * *$ \\
\hline Leg & $28.67 \pm 5.21$ & $36.94 \pm 9.76$ & $39.63 \pm 10.84$ & $0.000 * *$ & 0.457 (ns) & $0.000 * *$ \\
\hline \multicolumn{7}{|l|}{ Group 2} \\
\hline Forehead & $28.93 \pm 4.18$ & $33.19 \pm 7.86$ & $35.65 \pm 8.33$ & 0.617 (ns) & 0.720 (ns) & 0.224 (ns) \\
\hline Zygomatic & $27.50 \pm 4.53$ & $32.67 \pm 8.38$ & $35.56 \pm 9.23$ & 0.437 (ns) & 0.345 (ns) & 0.110 (ns) \\
\hline Hand & $34.15 \pm 8.59$ & $36.72 \pm 11.10$ & $36.70 \pm 11.25$ & 0.315 (ns) & $\mathrm{I} .000$ (ns) & 0.344 (ns) \\
\hline Forearm & $23.94 \pm 6.34$ & $23.58 \pm 4.06$ & $23.90 \pm 4.53$ & $\mathrm{I} .000$ (ns) & $\mathrm{I} .000$ (ns) & 1.000 (ns) \\
\hline Leg & $31.01 \pm 13.12$ & $33.25 \pm 10.75$ & $34.86 \pm 13.54$ & 0.614 (ns) & $\mathrm{I} .000$ (ns) & 0.571 (ns) \\
\hline
\end{tabular}

Notes: Surface and deep hydration values were obtained with different Moisturemeter frequency probes and are expressed in arbitrary units. $* P<0.05$, $* * P<0.001$. Abbreviations: ns, not significant; SD, standard deviation; T0, day 0; TI, day 15; T2, day 30; vs, versus. 
Table 3 Descriptive data (mean \pm SD) from relevant skin biomechanical descriptors and the respective comparative statistics obtained at the beginning (TO), after two weeks (TI), and at the end (T2) of the study

\begin{tabular}{|c|c|c|c|c|c|c|}
\hline & \multirow[t]{2}{*}{ T0 $($ mean \pm SD $)$} & \multirow[t]{2}{*}{ TI (mean \pm SD) } & \multirow[t]{2}{*}{ T2 (mean \pm SD) } & \multicolumn{3}{|l|}{$P$-values } \\
\hline & & & & (TI vs T0) & (T2 vs TI) & (T2 vs T0) \\
\hline \multicolumn{7}{|l|}{$U_{f}(\mathrm{~mm})$} \\
\hline \multicolumn{7}{|l|}{ Group I } \\
\hline Leg & $0.230 \pm 0.15$ & $0.195 \pm 0.16$ & $0.645 \pm 0.72$ & 0.305 (ns) & $0.003^{* *}$ & $0.007 * *$ \\
\hline Forearm & $0.815 \pm 0.42$ & $0.742 \pm 0.42$ & $1.173 \pm 0.70$ & $0.083(\mathrm{~ns})$ & $0.004 * *$ & $0.021 *$ \\
\hline Hand & $0.94 I \pm 0.43$ & $0.816 \pm 0.40$ & $1 .|73 \pm 0.7|$ & $0.509(\mathrm{~ns})$ & $0.56 \mathrm{I}(\mathrm{ns})$ & $0.004 * *$ \\
\hline Zygomatic & $1.798 \pm 0.67$ & $2.009 \pm 0.75$ & $1.889 \pm 0.75$ & $0.500(\mathrm{~ns})$ & $0.837(\mathrm{~ns})$ & $1.000(\mathrm{~ns})$ \\
\hline Forehead & $0.614 \pm 0.36$ & $0.706 \pm 0.86$ & $1.006 \pm 0.86$ & $0.45 \mathrm{I}(\mathrm{ns})$ & 0.169 (ns) & $0.03 I^{*}$ \\
\hline \multicolumn{7}{|l|}{ Group 2} \\
\hline Leg & $0.223 \pm 0.12$ & $0.188 \pm 0.11$ & $0.559 \pm 0.64$ & 0.934 (ns) & $0.352(\mathrm{~ns})$ & 0.492 (ns) \\
\hline Forearm & $0.772 \pm 0.22$ & $0.71 I \pm 0.23$ & $1.272 \pm 0.66$ & $\mathrm{I} .000(\mathrm{~ns})$ & 0.171 (ns) & $0.223(\mathrm{~ns})$ \\
\hline Hand & $0.867 \pm 0.16$ & $0.826 \pm 0.54$ & $1.196 \pm 0.72$ & $\mathrm{I} .000(\mathrm{~ns})$ & $0.322(\mathrm{~ns})$ & 0.395 (ns) \\
\hline Zygomatic & $1.594 \pm 0.77$ & $1.735 \pm 0.91$ & $1.711 \pm 0.89$ & $\mathrm{I} .000$ (ns) & $1.000(\mathrm{~ns})$ & $1.000(\mathrm{~ns})$ \\
\hline Forehead & $1.005 \pm 0.68$ & $0.872 \pm 0.70$ & $1.067 \pm 0.83$ & $\mathrm{I} .000(\mathrm{~ns})$ & $\mathrm{I} .000(\mathrm{~ns})$ & 1.000 (ns) \\
\hline \multicolumn{7}{|l|}{$U_{f}-U_{a}(m m)$} \\
\hline \multicolumn{7}{|l|}{ Group I } \\
\hline Leg & $0.032 \pm 0.02$ & $0.036 \pm 0.03$ & $0.774 \pm 0.59$ & $\mathrm{I} .000$ (ns) & $0.001 * * *$ & $0.001 * * *$ \\
\hline Forearm & $0.105 \pm 0.06$ & $0.093 \pm 0.06$ & $0.805 \pm 0.68$ & $0.364(\mathrm{~ns})$ & $0.001 * * *$ & $0.001 * * *$ \\
\hline Hand & $0.318 \pm 0.22$ & $0.247 \pm 0.13$ & $0.826 \pm 0.77$ & $\mathrm{I} .000(\mathrm{~ns})$ & $0.014 *$ & $0.012^{*}$ \\
\hline Zygomatic & $1.024 \pm 0.65$ & $1.323 \pm 0.83$ & $1.235 \pm 0.97$ & $0.380(\mathrm{~ns})$ & $1.000(\mathrm{~ns})$ & $0.819(\mathrm{~ns})$ \\
\hline Forehead & $0.183 \pm 0.11$ & $0.263 \pm 0.19$ & $0.758 \pm 0.56$ & $0.852(\mathrm{~ns})$ & $0.018^{*}$ & $0.030 *$ \\
\hline \multicolumn{7}{|l|}{ Group 2} \\
\hline Leg & $0.032 \pm 0.02$ & $0.022 \pm 0.01$ & $0.469 \pm 0.46$ & 0.403 (ns) & $0.522(\mathrm{~ns})$ & 0.549 (ns) \\
\hline Forearm & $0.121 \pm 0.06$ & $0.087 \pm 0.03$ & $0.814 \pm 0.80$ & $0.255(\mathrm{~ns})$ & $0.226(\mathrm{~ns})$ & 0.267 (ns) \\
\hline Hand & $0.163 \pm 0.13$ & $0.368 \pm 0.30$ & $0.899 \pm 0.89$ & $0.975(\mathrm{~ns})$ & $0.546(\mathrm{~ns})$ & 0.252 (ns) \\
\hline Zygomatic & $0.709 \pm 0.53$ & $1.26 \mathrm{I} \pm 0.88$ & $1.23 \mathrm{I} \pm 0.97$ & $0.484(\mathrm{~ns})$ & $1.000(\mathrm{~ns})$ & $0.490(\mathrm{~ns})$ \\
\hline Forehead & $0.65 I \pm 0.54$ & $0.525 \pm 0.55$ & $0.845 \pm 0.85$ & $1.000(\mathrm{~ns})$ & $0.690(\mathrm{~ns})$ & 1.000 (ns) \\
\hline \multirow{2}{*}{\multicolumn{7}{|c|}{$\begin{array}{l}U_{\mathrm{a}} / U_{\mathrm{f}} \\
\text { Group I }\end{array}$}} \\
\hline & & & & & & \\
\hline Leg & $0.838 \pm 0.06$ & $0.826 \pm 0.09$ & $0.604 \pm 0.35$ & 1.000 (ns) & $0.002 * *$ & $0.001 * *$ \\
\hline Forearm & $0.820 \pm 0.14$ & $0.809 \pm 0.13$ & $0.644 \pm 0.32$ & $\mathrm{I} .000(\mathrm{~ns})$ & $0.020 *$ & $0.008 * *$ \\
\hline Hand & $0.782 \pm 0.21$ & $0.776 \pm 0.18$ & $0.647 \pm 0.29$ & $\mathrm{I} .000(\mathrm{~ns})$ & $0.086(\mathrm{~ns})$ & $0.017^{*}$ \\
\hline Zygomatic & $0.577 \pm 0.27$ & $0.505 \pm 0.27$ & $0.595 \pm 0.32$ & $0.624(\mathrm{~ns})$ & 0.134 (ns) & 1.000 (ns) \\
\hline Forehead & $0.746 \pm 0.14$ & $0.727 \pm 0.19$ & $0.590 \pm 0.29$ & $1.000(\mathrm{~ns})$ & $0.044^{*}$ & $0.015^{*}$ \\
\hline \multicolumn{7}{|l|}{ Group 2} \\
\hline Leg & $0.824 \pm 0.07$ & $0.838 \pm 0.08$ & $0.655 \pm 0.30$ & $\mathrm{I} .000(\mathrm{~ns})$ & 0.406 (ns) & $0.365(\mathrm{~ns})$ \\
\hline Forearm & $0.790 \pm 0.08$ & $0.795 \pm 0.06$ & $0.607 \pm 0.31$ & $1.000(\mathrm{~ns})$ & $0.316(\mathrm{~ns})$ & $0.280(\mathrm{~ns})$ \\
\hline Hand & $0.765 \pm 0.15$ & $0.723 \pm 0.20$ & $0.617 \pm 0.33$ & 0.756 (ns) & 0.907 (ns) & 0.666 (ns) \\
\hline Zygomatic & $0.626 \pm 0.26$ & $0.543 \pm 0.23$ & $0.591 \pm 0.30$ & $1.000(\mathrm{~ns})$ & $1.000(\mathrm{~ns})$ & 1.000 (ns) \\
\hline Forehead & $0.612 \pm 0.22$ & $0.680 \pm 0.26$ & $0.610 \pm 0.32$ & $\mathrm{I} .000(\mathrm{~ns})$ & $0.847(\mathrm{~ns})$ & $1.000(\mathrm{~ns})$ \\
\hline \multirow{2}{*}{\multicolumn{7}{|c|}{$\begin{array}{l}U_{r} / U_{e} \\
\text { Group I }\end{array}$}} \\
\hline & & & & & & \\
\hline Leg & $\mathrm{I} .20 \mathrm{I} \pm 0.29$ & $|.300 \pm 0.3|$ & $0.925 \pm 0.63$ & $0.539(\mathrm{~ns})$ & $0.011^{*}$ & $0.05 \mathrm{I}(\mathrm{ns})$ \\
\hline Forearm & $0.860 \pm 0.28$ & $0.823 \pm 0.22$ & $0.622 \pm 0.38$ & $1.000(\mathrm{~ns})$ & $0.026^{*}$ & $0.006 * *$ \\
\hline Hand & $0.744 \pm 0.37$ & $0.768 \pm 0.33$ & $0.590 \pm 0.35$ & $\mathrm{I} .000$ (ns) & 0.068 (ns) & 0.084 (ns) \\
\hline Zygomatic & $0.403 \pm 0.24$ & $0.328 \pm 0.25$ & $0.385 \pm 0.26$ & $0.476(\mathrm{~ns})$ & 0.402 (ns) & 1.000 (ns) \\
\hline Forehead & $0.738 \pm 0.22$ & $0.7 \mid 2 \pm 0.27$ & $0.619 \pm 0.44$ & $\mathrm{I} .000(\mathrm{~ns})$ & $0.715(\mathrm{~ns})$ & $0.410(\mathrm{~ns})$ \\
\hline \multicolumn{7}{|l|}{ Group 2} \\
\hline Leg & $1.166 \pm 0.24$ & $1.230 \pm 0.43$ & $0.926 \pm 0.53$ & 1.000 (ns) & $0.406(\mathrm{~ns})$ & 0.365 (ns) \\
\hline Forearm & $0.765 \pm 0.17$ & $0.792 \pm 0.10$ & $0.529 \pm 0.34$ & $1.000(\mathrm{~ns})$ & 0.211 (ns) & $0.288(\mathrm{~ns})$ \\
\hline Hand & $0.72 I \pm 0.3 \mathrm{I}$ & $0.713 \pm 0.38$ & $0.6|5 \pm 0.4|$ & $\mathrm{I} .000(\mathrm{~ns})$ & $1.000(\mathrm{~ns})$ & $1.000(\mathrm{~ns})$ \\
\hline Zygomatic & $0.453 \pm 0.31$ & $0.326 \pm 0.21$ & $0.443 \pm 0.29$ & $0.670(\mathrm{~ns})$ & $0.446(\mathrm{~ns})$ & 1.000 (ns) \\
\hline Forehead & $0.57 I \pm 0.29$ & $0.654 \pm 0.33$ & $0.606 \pm 0.42$ & $\mathrm{I} .000$ (ns) & 1.000 (ns) & 1.000 (ns) \\
\hline
\end{tabular}


Table 3 (Continued)

\begin{tabular}{|c|c|c|c|c|c|c|}
\hline & \multirow[t]{2}{*}{ T0 $($ mean \pm SD $)$} & \multirow[t]{2}{*}{ TI (mean \pm SD) } & \multirow[t]{2}{*}{ T2 (mean \pm SD) } & \multicolumn{3}{|l|}{$P$-values } \\
\hline & & & & (TI vs T0) & (T2 vs TI) & (T2 vs T0) \\
\hline \multicolumn{7}{|l|}{$U_{v} / U_{e}$} \\
\hline \multicolumn{7}{|l|}{ Group I } \\
\hline Leg & $0.963 \pm 0.26$ & $1.081 \pm 0.32$ & $0.797 \pm 0.57$ & 0.277 (ns) & $0.040 *$ & 0.280 (ns) \\
\hline Forearm & $0.624 \pm 0.19$ & $0.631 \pm 0.16$ & $0.463 \pm 0.27$ & $\mathrm{I} .000(\mathrm{~ns})$ & $0.012^{*}$ & $0.015^{*}$ \\
\hline Hand & $0.576 \pm 0.33$ & $0.615 \pm 0.26$ & $0.46 I \pm 0.26$ & $\mathrm{I} .000(\mathrm{~ns})$ & $0.065(\mathrm{~ns})$ & $0.307(\mathrm{~ns})$ \\
\hline Zygomatic & $0.260 \pm 0.18$ & $0.215 \pm 0.21$ & $0.23 I \pm 0.16$ & 0.995 (ns) & $1.000(\mathrm{~ns})$ & 1.000 (ns) \\
\hline Forehead & $0.689 \pm 0.24$ & $0.661 \pm 0.26$ & $0.589 \pm 0.56$ & $1.000(\mathrm{~ns})$ & $1.000(\mathrm{~ns})$ & $0.966(\mathrm{~ns})$ \\
\hline \multicolumn{7}{|l|}{ Group 2} \\
\hline Leg & $0.979 \pm 0.24$ & $1.05 I \pm 0.39$ & $0.864 \pm 0.54$ & 1.000 (ns) & $\mathrm{I} .000$ (ns) & 1.000 (ns) \\
\hline Forearm & $0.630 \pm 0.20$ & $0.686 \pm 0.15$ & $0.442 \pm 0.25$ & $0.640(\mathrm{~ns})$ & $0.080(\mathrm{~ns})$ & $0.166(\mathrm{~ns})$ \\
\hline Hand & $0.544 \pm 0.12$ & $0.588 \pm 0.23$ & $0.490 \pm 0.28$ & $\mathrm{I} .000(\mathrm{~ns})$ & $0.777(\mathrm{~ns})$ & $1.000(\mathrm{~ns})$ \\
\hline Zygomatic & $0.368 \pm 0.24$ & $0.309 \pm 0.23$ & $0.334 \pm 0.29$ & 1.000 (ns) & $1.000(\mathrm{~ns})$ & $1.000(\mathrm{~ns})$ \\
\hline Forehead & $0.56 I \pm 0.24$ & $0.606 \pm 0.32$ & $0.535 \pm 0.42$ & $\mathrm{I} .000(\mathrm{~ns})$ & $1.000(\mathrm{~ns})$ & $1.000(\mathrm{~ns})$ \\
\hline
\end{tabular}

Notes: $* P<0.05, * * P<0.001$.

Abbreviations: ns, not significant; SD, standard deviation; T0, day 0 ; TI, day 15 ; T2, day 30 ; $U_{f}$, extensibility; $U_{f}-U_{a}$, the ability of the skin to return to its original state; $U_{a}$, $U_{f}$, total elasticity (including pulse stretching and recovery); $U_{r} / U_{e}$, elastic function; $U_{v} / U_{e}$, viscoelasticity; vs, versus.

\section{Statistics}

Statistical analysis (descriptive and comparative) was performed using the SPSS version 20.0 (SPSS Inc., Chicago, IL, USA) software. The cluster's analysis, which is based on a hierarchic model that groups similar "objects", allowed to identify a group of individuals consuming less than $3,200 \mathrm{~mL} /$ day $(\mathrm{n}=38)$ as Group 1, and another group of individuals consuming more than $3,200 \mathrm{~mL} /$ day $(\mathrm{n}=11)$, as Group 2. The analysis of variance generalized linear model, the sphericity test of Mauchly, and the Levene test were applied to both groups. The goal was to test the homogeneity of these populations. So, we confirmed that variance of endpoint variables for these two groups were similar and that the data distribution was narrow, tending to normal. Post hoc multiple comparisons were performed using the Bonferroni test, a suitably robust method to control errors in handling. ${ }^{30}$ Variables were also compared by the Spearman correlation coefficient. A confidence level of $95 \%$ was adopted. Due to the population homogeneity of age, age correction was not regarded as necessary.

\section{Results and discussion}

The water consumption pattern was crucial to assess the impact of dietary water on the cutaneous physiology within this population. Previous results have drawn attention to the importance of $\mathrm{DiW}$ in quantitative terms, representing more than $50 \%$ of the total water related to diet. ${ }^{1,21,31} \mathrm{DiW}$ includes water from food, juices and soup, and in accordance with those results, our FFQ detected that DiW contributed 57.2\% of the total water accounted within these patients' diets, stressing the need to consider all sources of water input to calculate the total water consumption (Figure 1).

Results obtained from both groups having different daily water consumptions revealed no relevant changes regarding epidermal barrier and TEWL. As shown in Table 1, we found a progressive decreasing gradient from the face, the highest (forehead and zygomatic area), to the forearm and leg, where the lowest values were recorded in both groups. This is in accord with the known expected anatomical and functional variation. ${ }^{32-35}$ Thus, our methodology did not significantly change the epidermal barrier in both groups. Regarding epidermal hydration, however, a dramatically different reality was registered, with a consistent improvement of superficial and deep hydration in both groups, although with different magnitudes (Table 2). In fact, changes observed in the group with lower initial water consumption (Group 1) were significantly greater and present in all anatomical areas, relative to the (reduced) impact observed in Group 2. Similar results were previously reported in individuals with dry skin, leading the authors to suggest that increasing the dietary water intake would affect the skin the same way as a topical moisturizer. ${ }^{2}$ In our current study, impact on epidermal hydration was consistently noticed in both surface and deep hydration variables, which may signify that more water is available for the normal physiological processes (Table 2). These effects are especially detectable in Group 1, from T1 forward.

In order to look further into the impact of this dietary water overload on the skin's physiology, we also assessed the so-called "envelope" function. Skin hydration has been related to skin mechanics to justify preservation of a younger, 
Table 4 Correlations (Spearman) found between epidermal hydration variables (superficial and deep) and the most relevant biomechanical descriptors obtained in the beginning (T0), after 2 weeks (TI) and in the end (T2) of the study

\begin{tabular}{|c|c|c|c|c|c|c|}
\hline & \multicolumn{2}{|l|}{ T0 } & \multicolumn{2}{|l|}{ TI } & \multicolumn{2}{|l|}{ T2 } \\
\hline & $\boldsymbol{R}$ & $P$-value & $R$ & $P$-value & $\boldsymbol{R}$ & $P$-value \\
\hline \multicolumn{7}{|l|}{ Face } \\
\hline \multicolumn{7}{|c|}{ Group I } \\
\hline \multicolumn{7}{|c|}{ Surface hydration } \\
\hline$U_{f}$ & -0.113 & 0.489 (ns) & 0.005 & 0.976 (ns) & 0.405 & $0.010 *$ \\
\hline$U_{f}-U_{a}$ & -0.004 & $0.980(\mathrm{~ns})$ & 0.382 & $0.016^{*}$ & 0.567 & $0.000 * * *$ \\
\hline$U_{a} / U_{f}^{d}$ & 0.021 & $0.90 \mathrm{I}(\mathrm{ns})$ & 0.011 & $0.945(\mathrm{~ns})$ & -0.132 & 0.425 (ns) \\
\hline \multicolumn{7}{|c|}{ Deep hydration } \\
\hline$U_{f}$ & -0.036 & 0.835 (ns) & -0.053 & $0.76 \mathrm{I}$ (ns) & 0.296 & $0.07 \mathrm{I}(\mathrm{ns})$ \\
\hline$U_{f}-U_{a}$ & -0.038 & 0.827 (ns) & 0.342 & $0.044^{*}$ & 0.528 & $0.00 I^{* * *}$ \\
\hline$U_{a} / U_{f}$ & -0.096 & $0.56 \mathrm{I}(\mathrm{ns})$ & -0.120 & $0.466(\mathrm{~ns})$ & -0.75 & 0.648 (ns) \\
\hline \multicolumn{7}{|c|}{ Group 2} \\
\hline \multicolumn{7}{|c|}{ Surface hydration } \\
\hline$U_{f}$ & 0.722 & $0.018^{*}$ & 0.684 & $0.029 *$ & 0.636 & $0.048 *$ \\
\hline$U_{f}^{f}-U_{a}$ & 0.043 & 0.907 (ns) & 0.394 & $0.260(\mathrm{~ns})$ & 0.600 & 0.067 (ns) \\
\hline$U_{a}^{f} / U_{f}^{a}$ & -0.597 & 0.068 (ns) & -0.264 & 0.462 (ns) & $-0.85 I$ & $0.002 * *$ \\
\hline \multicolumn{7}{|c|}{ Deep hydration } \\
\hline$U_{f}$ & -0.422 & 0.258 (ns) & 0.540 & 0.133 (ns) & 0.576 & $0.082(\mathrm{~ns})$ \\
\hline$U_{f}-U_{a}$ & -0.600 & $0.088(\mathrm{~ns})$ & 0.583 & $0.099(\mathrm{~ns})$ & 0.709 & $0.002 *$ \\
\hline$U_{a} / U_{f}$ & 0.273 & $0.084(\mathrm{~ns})$ & -0.487 & 0.153 (ns) & -0.505 & 0.137 (ns) \\
\hline \multicolumn{7}{|l|}{ Upper limb } \\
\hline \multicolumn{7}{|c|}{ Group I } \\
\hline \multicolumn{7}{|c|}{ Surface hydration } \\
\hline$U_{f}$ & -0.447 & $0.004^{* *}$ & -0.060 & $0.720(\mathrm{~ns})$ & 0.005 & 0.977 (ns) \\
\hline$U_{f}-U_{a}$ & -0.138 & 0.394 (ns) & $-0.27 \mid$ & 0.095 (ns) & 0.125 & $0.460(\mathrm{~ns})$ \\
\hline$U_{a} / U_{f}^{d}$ & 0.205 & 0.200 (ns) & 0.347 & $0.030 *$ & 0.097 & 0.556 (ns) \\
\hline \multicolumn{7}{|c|}{ Deep hydration } \\
\hline$U_{f}$ & -0.294 & $0.086(\mathrm{~ns})$ & -0.276 & $0.114(\mathrm{~ns})$ & -0.014 & 0.936 (ns) \\
\hline$U_{f}-U_{a}$ & 0.006 & $0.973(\mathrm{~ns})$ & -0.426 & $0.011 *$ & 0.381 & $0.020 *$ \\
\hline$U_{a} / U_{f}$ & -0.072 & $0.664(\mathrm{~ns})$ & 0.173 & $0.292(\mathrm{~ns})$ & -0.099 & 0.549 (ns) \\
\hline \multicolumn{7}{|c|}{ Group 2} \\
\hline \multicolumn{7}{|c|}{ Surface hydration } \\
\hline$U_{f}$ & -0.468 & 0.173 (ns) & -0.721 & $0.019 *$ & 0.072 & $0.865(\mathrm{~ns})$ \\
\hline$U_{f}-U_{a}$ & -0.390 & 0.265 (ns) & 0.188 & $0.603(\mathrm{~ns})$ & 0.758 & $0.01 I^{*}$ \\
\hline$U_{a} / U_{f}^{a}$ & -0.238 & $0.508(\mathrm{~ns})$ & 0.131 & $0.719(\mathrm{~ns})$ & -0.775 & $0.008^{* *}$ \\
\hline Deep hydr & & & & & & \\
\hline$U_{f}$ & -0.157 & 0.687 (ns) & -0.750 & $0.020 *$ & -0.206 & $0.624(\mathrm{~ns})$ \\
\hline$U_{f}-U_{a}$ & -0.305 & 0.424 (ns) & -0.400 & $0.286(\mathrm{~ns})$ & 0.923 & $0.000 * * *$ \\
\hline$U_{a} / U_{f}$ & 0.534 & $0.112(\mathrm{~ns})$ & 0.467 & 0.173 (ns) & -0.742 & $0.014 *$ \\
\hline Leg & & & & & & \\
\hline Group I & & & & & & \\
\hline Surface hy & & & & & & \\
\hline$U_{f}$ & -0.373 & $0.019 *$ & -0.356 & $0.028^{*}$ & -0.179 & $0.295(\mathrm{~ns})$ \\
\hline$U_{f}-U_{a}$ & -0.468 & $0.002^{* *}$ & -0.476 & $0.003^{* *}$ & 0.111 & $0.5 \mathrm{II}$ (ns) \\
\hline$U_{a} / U_{f}^{d}$ & -0.165 & 0.309 (ns) & 0.097 & $0.553(\mathrm{~ns})$ & -0.109 & 0.503 (ns) \\
\hline Deep hydr & & & & & & \\
\hline$U_{f}$ & -0.266 & 0.123 (ns) & 0.099 & 0.578 (ns) & -0.148 & 0.389 (ns) \\
\hline$U_{f}-U_{a}$ & -0.050 & $0.765(\mathrm{~ns})$ & -0.121 & 0.496 (ns) & 0.216 & 0.199 (ns) \\
\hline$U_{a} / U_{f}$ & -0.049 & $0.763(\mathrm{~ns})$ & 0.209 & 0.196 (ns) & -0.173 & 0.285 (ns) \\
\hline Group 2 & & & & & & \\
\hline Surface hy & & & & & & \\
\hline$U_{f}$ & -0.292 & 0.414 (ns) & 0.006 & 0.987 (ns) & 0.095 & 0.824 (ns) \\
\hline$U_{f}-U_{a}$ & -0.455 & 0.187 (ns) & -0.103 & 0.777 (ns) & 0.723 & $0.018^{*}$ \\
\hline$U_{a} / U_{f}^{a}$ & 0.315 & $0.375(\mathrm{~ns})$ & 0.379 & $0.280(\mathrm{~ns})$ & 0.316 & $0.374(\mathrm{~ns})$ \\
\hline Deep hydr & & & & & & \\
\hline$U_{f}$ & -0.496 & 0.174 (ns) & -0.117 & 0.765 (ns) & 0.176 & 0.678 (ns) \\
\hline$U_{f}-U_{a}$ & -0.517 & 0.154 (ns) & -0.200 & $0.606(\mathrm{~ns})$ & 0.496 & 0.145 (ns) \\
\hline$U_{a} / U_{f}^{a}$ & 0.111 & $0.760(\mathrm{~ns})$ & 0.102 & $0.779(\mathrm{~ns})$ & -0.212 & 0.556 (ns) \\
\hline
\end{tabular}

Notes: $* P<0.05, * * P<0.01, * * * P<0.001$.

Abbreviations: ns, not significant; T0, day 0; TI, day $15 ; \mathrm{T} 2$, day $30 ; U_{\mathrm{f}}$ extensibility; $U_{\mathrm{f}}-U_{\mathrm{a}^{\prime}}$, the ability of the skin to return to its original state; $U_{\mathrm{a}} / U_{\mathrm{f}}$ total elasticity (including pulse stretching and recovery); $U_{\mathrm{r}} / U_{\mathrm{e}}$, elastic function; $U_{\mathrm{v}} / U_{\mathrm{e}}$, viscoelasticity. 
healthier looking skin. Dermal water was reported to decrease the friction between fibers, acting as a "lubricant", including in the upper layers, thus facilitating the dynamics of the overall structure. ${ }^{26,36}$ However, a direct relationship between skin hydration and biomechanics has not been clearly demonstrated. ${ }^{37-39}$

Biomechanical descriptors such as maximum extensibility $\left(U_{\mathrm{f}}\right)$, the ability to return to the original state $\left(U_{\mathrm{f}}-U_{\mathrm{a}}\right)$, total elasticity $\left(U_{\mathrm{a}} / U_{\mathrm{f}}\right)$, elastic function $\left(U_{\mathrm{r}} / U_{\mathrm{e}}\right)$, and the viscoelastic ratio $\left(U_{\mathrm{v}} / U_{\mathrm{e}}\right)$ were chosen as the most relevant, in accordance to several authors ${ }^{40,41}$ and because they have been referred as the most useful to detect improvements in the plasticity of skin $^{41-45}$ (Table 3). As mentioned previously, our experimental methodology did impact skin biomechanics in both groups with different magnitudes, and statistically significant evidence of biomechanical changes could be found in Group 1 (Table 3$)$. Total extensibility $\left(U_{\mathrm{f}}\right)$ significantly improved in all body areas except the face after the 2 weeks of the test. By the end of the study, these improvements were still present in all the tested regions, with significant differences present in the leg, forearm, hand, and in the forehead (Table 3). Similar impact was registered for the ability of the skin to return to its original state $\left(U_{\mathrm{f}}-U_{\mathrm{a}}\right)$, which significantly increased after the 2 weeks (T1) and 4 weeks (T2) of the water surplus in all the tested areas, except the zygomatic region in the face. This consistent increase of $U_{\mathrm{f}}$ and $U_{\mathrm{f}}-U_{\mathrm{a}}$ throughout the study seems to be related with the highest amount of water available in the epidermis of these volunteers, facilitating deformation and recovery after stress, as has been previously suggested. ${ }^{25,46}$ The evolution of the other ratios are more difficult to follow. Total elasticity $\left(U_{\mathrm{a}} / U_{\mathrm{f}}\right)$ was significantly reduced in all tested areas except the zygomatic, especially in $\mathrm{T} 2$, and this reflects a higher impact of the water surplus on skin extensibility (Table 3 ) rather than in the elastic recovery $\left(U_{\mathrm{a}}\right)$. The elastic function $U_{\mathrm{r}} / U_{\mathrm{e}}$ and viscoelasticity $\left(U_{\mathrm{v}} / U_{\mathrm{e}}\right)$ do not follow this pattern. However, these descriptors are closely age-related, thus they depend primarily on the dermal components whose contribution(s) cannot be specifically quantified with these methods. ${ }^{28,40,47,48} \mathrm{~A}$ decrease in the viscoelasticity index has been reported after regular long-term use of topical moisturizers..$^{26,43}$

We have finally analyzed a potential relationship between the most relevant descriptors representing epidermal hydration and biomechanics. In fact, many of the factors that modify skin mechanical properties have been identified, but relationships between epidermal structure and these characteristics are still insufficiently documented. Although suggested for many years, ${ }^{49}$ experimental evidence is still recent (although rare), and are frequently obtained from other perspectives. ${ }^{50-52}$ Moreover, the in vivo approach is particularly difficult, considering the close relationship between cutaneous tissues and the poor discriminative capacity of currently available technology. Recent results from a three-layer computational skin model ${ }^{53}$ and from a new dynamic mechanical device, ${ }^{54}$ both assessing mechanical properties of skin under different conditions, suggested that the epidermis, the statum corneum in particular, and different factors such as hydration, do influence skin mechanical properties in vivo as well.

After calculating a confidence range for each anatomical area, our data was tested by the Spearman correlation coefficient, a well-known determinant for these properties. ${ }^{41,45}$ In this way, an estimated interval allowed a mean value calculation for each variable in the face, upper limb (forearm and hand), and leg in both groups. As shown in Table 4, clear, consistent relationships between epidermal hydration and biomechanical descriptors could not be found under the present experimental conditions. Nevertheless, significant relationships are nearly absent in both groups at $\mathrm{T} 0$. The water stress test seems to contribute to the significant relationships found, especially at T2 and in particular within Group 1. The progressive reduction of these relationships from the face to the leg areas should also be mentioned (Table 4). More sensitive, discriminative technology may be a key aspect behind this apparent absence of differential data.

\section{Conclusion}

The clinical relevance of this approach should be strongly emphasized in specific conditions where the correction of skin water balance by strategies other than pharmacological might dramatically improve the patient's quality of life. This particularly may be the case for the elderly or obese, for whom dry skin is a consistent compliant.

The present methodology allowed, for the first time, an objective clinical approach to study the effects of dietary water on normal skin physiology. These results seem to confirm that higher water inputs in one's regular diet might positively impact normal skin physiology, as expressed by its hydration and biomechanical behavior, and in particular in those individuals with lower daily water consumptions.

\section{Acknowledgments}

The authors thanks all patients enrolled in the study, and Carla Monteiro and Osvaldo Santos for statistical support.

\section{Disclosure}

The authors report no conflicts of interest in this work. 


\section{References}

1. Palma ML, Monteiro C, Tavares L, Julia M, Rodrigues LM. Relationship between the dietary intake of water and skin hydration. Biomed Biopharm Res. 2012;9:173-181.

2. Mac-Mary S, Creidi P, Marsaut D, et al. Assessment of effects of an additional dietary natural mineral water uptake on skin hydration in healthy subjects by dynamic barrier function measurements and clinic scoring. Skin Res Technol. 2006;12:199-205.

3. Palma L, Tavares L, Bujan MRL. Diet related water seems to affect in vivo skin hydration and biomechanics. IUPS 2013 [Abstract B]. 2013:801. Available from: http://edition.pagesuite-professional.co.uk// launch.aspx?eid=3a1c456e-d4f7-4725-98f6-b1d998184a5e. Accessed June 17, 2015.

4. Benelam B, Wyness L. Hydration and health: a review. Nutr Bull. 2010;35:3-25.

5. Arnaud MJ, Noakes TD. Should humans be encouraged to drink water to excess? Eur J Clin Nutr.; 2011;65:875-876.

6. Häussinger D. The role of cellular hydration in the regulation of cell function. Biochem J. 1996;313:697-710.

7. Lang F, Busch GL, Ritter M, et al. Functional significance of cell volume regulatory mechanisms. Physiol Rev. 1998;78:247-306.

8. Grandjean A, Campbell S. Hydration: Fluids for Life. Washington, DC: The International Life Science Institute; 2004:50.

9. Ritz P, Berrut G. The importance of good hydration for day-to-day health. Nutr Rev. 2005;63:S6-S13.

10. Popkin BM, Rosenberg IH. Water, hydration and health. Nutr Rev. 2010;439-458.

11. Szinnai G, Schachinger H, Arnaud MJ, Linder L, Keller U. Effect of water deprivation on cognitive-motor performance in healthy men and women. Am J Physiol Regul Integr Comp Physiol. 2005;289: R275-R280.

12. Jéquier $\mathrm{E}$, Constant $\mathrm{F}$. Water as an essential nutrient: the physiological basis of hydration. Eur J Clin Nutr. 2010;64:115-123.

13. Sawka MN, Cheuvront SN, Iii RC. Human water needs. Nutr Rev. 2005;63:S30-S39.

14. Thornton SN. Thirst and hydration: physiology and consequences of dysfunction. Physiol Behav. 2010;100:15-21.

15. US Department of Agriculture and US Department of Health and Human Services. Dietary Guidelines for Americans 2010. 7th ed. Washington, DC: US Government Printing Office; December 2010.

16. Panel E, Nda A. Scientific opinion on dietary reference values for water [Internet]. EFSA J. 2010;8(3):1-48. Available from: http://www.efsa. europa.eu/en/efsajournal/doc/1459.pdf. Accessed November 4, 2013.

17. Vergou T, Schanzer S, Richter H. Comparison between TEWL and laser scanning microscopy measurements for the in vivo characterization of the human epidermal barrier. J Biophotonics. 2012;5:152-158.

18. Rosado C, Rodrigues LM. In vivo study of the physiological impact of stratum corneum sampling methods. Int J Cosmet Sci. 2003;25: 37-44.

19. Rosado C, Pinto P, Rodrigues LM. Modeling TEWL-desorption curves: a new practical approach for the quantitative in vivo assessment of skin barrier. Exp Dermatol. 2005;14:386-390.

20. Williams S, Krueger N, Davids M, Kraus D, Kerscher M. Effect of fluid intake on skin physiology: distinct differences between drinking mineral water and tap water. Int J Cosmet Sci. 2007;29:131-138.

21. Wolf R, Wolf D, Rudikoff D, Parish LC. Nutrition and water: drinking eight glasses of water a day ensures proper skin hydration-myth or reality? Clin Dermatol. 2010;28:380-383.

22. Palma ML, Monteiro C, Bujan MJ, Rodrigues LM. Water intake and relationship with the water content of diet in a healthy population. Biomed Biopharm Res. 2011;8:261-271.

23. Rogiers V; EEMCO Group. EEMCO guidance for the assessment of transepidermal water loss in cosmetic sciences. Skin Pharmacol Appl Skin Physiol. 2001;14:117-128.

24. Krueger N, Luebberding S, Oltmer M, Streker M, Kerscher M. Agerelated changes in skin mechanical properties: a quantitative evaluation of 120 female subjects. Skin Res Technol. 2011;17:141-148.
25. Wilhelm KP, Cua AB, Maibach HI. Skin aging. Effect on transepidermal water loss, stratum corneum hydration, skin surface $\mathrm{pH}$, and casual sebum content. Arch Dermatol. 1991;127:1806-1809.

26. Mahmood T, Akhtar N, Khan BA, Shoaib Khan HM, Saeed T. Changes in skin mechanical properties after long-term application of cream containing green tea extract. Aging Clin Exp Res. 2010;23: 333-336.

27. Assembly G. Declaration of Helsinki. Ethical principles for medical research involving human subjects. J Indian Med Assoc. 2009;107: 403-405.

28. Hussain SH, Limthongkul B, Humphreys TR. The biomechanical properties of the skin. Dermatol Surg. 2013;39:193-203.

29. Ventre M, Mollica F, Netti PA. The effect of composition and microstructure on the viscoelastic properties of dermis. J Biomech. 2009;42: 430-435.

30. Field A. Discovering Statistics Using SPSS. London, UK: Sage Publications; 2009:166-181.

31. Panel on Dietary Reference Intakes for Electrolytes and Water. SC on the SE of DRI. DRI Dietary Reference Intakes for Water, Potassium, Sodium, Cloride and Sulfate. Washington, DC: Institute of Medicine of the National Academies; 2004:73.

32. Youn SW, Kim SJ, Hwang IA, Park KC. Evaluation of facial skin type by sebum secretion: Discrepancies between subjective descriptions and sebum secretion. Skin Res Technol. 2002;8:168-172.

33. Luebberding S, Krueger N, Kerscher M. Age-related changes in skin barrier function - quantitative evaluation of 150 female subjects. Int $J$ Cosmet Sci. 2013;35:183-190.

34. Marrakchi S, Maibach HI. Biophysical parameters of skin: map of human face, regional, and age-related differences. Contact Dermatitis. 2007;57:28-34.

35. Egawa M, Tagami H. Comparison of the depth profiles of water and water-binding substances in the stratum corneum determined in vivo by Raman spectroscopy between the cheek and volar forearm skin: effects of age, seasonal changes and artificial forced hydration. Br J Dermatol. 2008;158:251-260.

36. Kim BY, Choi JW, Park KC, Youn SW. Sebum, acne, skin elasticity, and gender difference - which is the major influencing factor for facial pores? Skin Res Technol. 2013;19:e45-e53.

37. Kapoor S, Saraf S. Assessment of viscoelasticity and hydration effect of herbal moisturizers using bioengineering techniques. Pharmacogn Mag. 2010;6:298-304.

38. Wissing SA, Müller RH. The influence of solid lipid nanoparticles on skin hydration and viscoelasticity - in vivo study. Eur J Pharm Biopharm. 2003;56:67-72.

39. Maresh CM, Gabaree-Boulant CL, Armstrong LE, et al. Effect of hydration status on thirst, drinking, and related hormonal responses during low-intensity exercise in the heat. J Appl Physiol. 2004;97: 39-44.

40. Luebberding S, Krueger N, Kerscher M. Mechanical properties of human skin in vivo: a comparative evaluation in 300 men and women. Skin Res Technol. 2014;20:127-135.

41. Cua AB, Wichelm K-P, Maibach HI. Frictional properties of human skin: relation to age, sex and anatomical region, stratum corneum hydration and transepidermal water loss. Br J Dermatol. 1990;123:473-479.

42. Jemec GB, Na R. Hydration and plasticity following long-term use of a moisturizer: a single-blind study. Acta Derm Venereol. 2002;82: $322-324$.

43. Akhtar N, Zaman SU, Khan BA, Amir MN, Ebrahimzadeh MA. Calendula extract: effects on mechanical parameters of human skin. Acta Pol Pharm. 2011;68:693-701.

44. Ryu HS, Joo YH, Kim SO, Park KC, Youn SW. Influence of age and regional differences on skin elasticity as measured by the Cutometer. Skin Res Technol. 2008;14:354-358.

45. Hendriks FM, Brokken D, Oomens CW, Bader DL, Baaijens FP. The relative contributions of different skin layers to the mechanical behavior of human skin in vivo using suction experiments. Med Eng Phys. 2006;28:259-266. 
46. Dobrev H. Use of Cutometer to assess epidermal hydration. Skin Res Technol. 2000;6:239-244.

47. Alexander H, Cook T. Variations with age in the mechanical properties of human skin in vivo. J Tissue Viability. 2006;16:6-11.

48. Ganceviciene R, Liakou AI, Theodoridis A, Makrantonaki E, Zouboulis CC. Skin anti-aging strategies. Dermatoendocrinol. 2012;4: 308-319.

49. De Rigal J, Leveque JL. In vivo measurement of stratum corneum elasticity. Bioeng Skin. 1985;1:13-23.

50. Jiang ZX, DeLaCruz J. Appearance benefits of skin moisturization. Skin Res Technol. 2011;17:51-55.

51. Ooe M, Tanida K, Yamamura T. Influence of hydration for optical properties of stratum corneum. J Soc Cosmet Chem Japan. 2010;2: 333-337.
52. Veijgen NK, van der Heide E, Masen MA. A multivariable model for predicting the frictional behaviour and hydration of the human skin. Skin Res Technol. 2013;19:330-338. doi: 0.1111/srt.12053.

53. Lévêque JL, Audoly B. Influence of Stratum Corneum on the entire skin mechanical properties, as predicted by a computational skin model. Skin Res Technol. 2013;19:42-46. doi: 10.1111/j.16000846.2012.00664.x.

54. Sandford E, Chen Y, Hunter I, Hillebrand G, Jones L. Capturing skin properties from dynamic mechanical analyses. Skin Res Technol. 2013;19(1):e339-e348. doi: 10.1111/j.1600-0846.2012.00649.

\section{Publish your work in this journal}

Clinical, Cosmetic and Investigational Dermatology is an international, peer-reviewed, open access, online journal that focuses on the latest clinical and experimental research in all aspects of skin disease and cosmetic interventions. All areas of dermatology will be covered; contributions will be welcomed from all clinicians and

Submit your manuscript here: http://www.dovepress.com/clinical-cosmetic-and-investigational-dermatology-journal basic science researchers globally. This journal is indexed on CAS. The manuscript management system is completely online and includes a very quick and fair peer-review system, which is all easy to use. Visit http://www.dovepress.com/testimonials.php to read real quotes from published authors. 